\title{
Model-free Adaptive Fuzzy Logic Control for a Half-car Active Suspension System
}

\author{
Ghazally I. Y. MUSTAFA ${ }^{1,2}$, Haoping WANG ${ }^{1 *}$, Yang TIAN ${ }^{1}$ \\ ${ }^{1}$ Sino-French International Joint Laboratory of Automatic Control and Signal Processing, School of \\ Automation, Nanjing University of Science and Technology Nanjing, 210094, China \\ hp.wang@njust.edu.cn (*Corresponding author) \\ ${ }^{2}$ Department of Electrical Engineering, Damazin Technical College, Sudan Technological University, Sudan
}

\begin{abstract}
This paper presents a novel model-free adaptive fuzzy logic controller (MFAFLC) for vibration control of active suspension systems. The physical constraint of the half-car model is considered so that the parameter's variation and external disturbance which are simultaneously taken into account may provide a realistic framework. The control objective is to deal with the classical conflict between minimizing both the vertical and angular chassis accelerations which increase the ride comfort. On the other side, the dynamic wheel and suspension deflection remain within safety limits to ensure the ride safety. The proposed scheme contains three parts: the first one is, the model-free based intelligent PI controller which overcomes the existing controller's complexity, introduces requisite performances, and reduces the output high order derivative. The second one is the extended state observer (ESO) which is utilized to estimate the model unknown uncertain dynamics. The third one, the adaptive fuzzy logic based fractional order control is used to compensate the ESO estimation error online. The feature of MFAFLC is a quite simple structure and easy to be implemented. The entire closed-loop system stability is proved based on the Lyapunov theory and the Barbalat's Lemma. Finally, to demonstrate the performance of the proposed MFAFLC, the comparison with the classical PID, and intelligent PID is conducted under three different road disturbances.
\end{abstract}

Keywords: Active suspension system, Half-car model, Model-free control, Adaptive fuzzy logic control, Extended state observer.

\section{Introduction}

Recently, automobile suspension systems have drawn much attention from both the researchers and the car industry due to their impact on the chassis performance of vehicles. Better vehicle suspension should ever provide adequate comfort, proper handling, and ability to maintain roadholding (Wang et al., 2018a). Nowadays, to guarantee the ride comfort and driving safety, a significant numbers of automotive suspension models have been developed as well as controller design approaches (Hrovat, 1997, Savaresi et al., 2010, Song, J., Niu, Y. and Zou, Y., 2018). Moreover, the suspension systems have an extensive impact on the subjective impression of the car (Sun et al., 2014). Thus, all the forces between the car body and the surface of the road are pass and are filtered through the suspension systems, hence substantially controlling the comfort and safety of the ride. Among the common current modes of suspension systems, the active suspension system is widely accepted despite its high-power consumption, because the control of this suspension type can effectively improve the suspension performance over both conventional passive and semi-active suspension systems (Cao et al., 2008, Chen et al., 2015). Therefore, many control methods are designed to ensure the suspension systems stability and performance enhancement, such as linear quadratic-Gaussian control (Chen et al., 2012), adaptive control (Pan et al., 2017, Chen and Huang, 2005), sliding mode control (Yoshimura et al., 2001, Sam et al., 2004, Pusadkar et al., 2019), fuzzy logic control (Cao, J., Li, P. and Liu, H, 2010, Rao and Prahlad, 1997), backstepping control (Pang, H., Zhang, X., and Xu, Z, 2018, Yagiz and Hacioglu, 2008).

In the active suspension system, the inevitable existence of the model's nonlinearities such as parameter changes and external perturbations may reduce both the ride comfort and safety. To solve this problem, several various control techniques have been proposed. It is well known that the sliding mode control has the ability to deal with uncertain systems. Thus, the sliding mode control is combined with other approaches which are introduced in detail in (Zhao et al., 2015, Moradi and Fekih, 2014). Moreover, intelligent control approaches such as fuzzy logic control, have confirmed the results of the robust closedloop systems (Demir, O., Keskin, I., and Cetin, S, 2012, Hafaifa, A., Laaouad, F., and Laroussi, K, 2010). A Takagi-Sugeno fuzzy logic based sliding-mode technique (Wen et al., 2017) and adaptive fuzzy logic based backstepping control has been implemented for the active suspension system to enhance the riding comfort and keep the riding safety within the safe operating limits (Sun et al., 2018). The feedback control design is reported in (Li, P., Lam, J., and Cheung, K. C, 
2014b, Wang, G., Chen, C. and Yu, S., 2016a, Li, P., Lam, J., and Cheung, K. C., 2014c). In (Li, P., Lam, J., and Cheung, K. C., 2014b), based on cone complementarity linearization algorithm and linear matrix inequality (LMI), the output-feedback signal is used in Control design. In this work, the ride quality is measured with $H_{\infty}$ norm approach, while the generalized $\mathrm{H}_{2}$ norm is utilized to deal with the hard constraints on the peak values of some variables. Furthermore, the output-feedback control approach based on the variable substitution and LMI are proposed for the active suspension model with constrained information (Wang, G., Chen, C. and Yu, S., 2016a).

Considering the above mentioned problem, most of the control procedures which have been discussed before might afford the best trajectory tracking for the linearized active suspension system, which requires an accurate model. However, this kind of approaches especially model-based one has various well-known limitations. Furthermore, these strategies need considerable calculations that may prevent them from being executed in real time. Moreover, for the active suspension system, the vehicle sprung, and unsprung masses are varying with the number of vehicle passengers and the payload. Nevertheless, this type of nonlinearities is not considered in the research works (Li, H., Jing, X., and Karimi, H. R., 2014b, Wang, G., Chen, C. and Yu, S., 2016a, Li, P., Lam, J., \& Cheung, K. C. , 2014c). As a result in (Li et al., 2013), if the variations of the vehicle sprung and unsprung masses are not considered in the control-design process, the performance of the vehicle suspension systems will be affected.

In order to enhance the performance tracking and to replace the mathematical model and the uncertainty, a new model-free approach based intelligent proportional-integral-derivative (iPID) has been introduced in (Fliess and Join, 2008). Furthermore, iPID is confirmed to control various systems such as wind turbine systems (Fliess and Join, 2013, Wang et al., 2015a, Li et al., 2016). Excellent results were achieved when integrating this method with other strategies in (Wang et al., 2016b, Han, S., Wang, H.P., and Tian, Y., 2018), for instance to confirm the robustness of the control synthesis the method is integrated with neural network control and time-delay estimation (Zhang et al., 2018) while in order to guarantee stability for the unknown nonlinear system the method is integrated with fractional order sliding mode control, and optimized fuzzy logic control, in (Mustafa et al., 2019, Wang, H.P., Mustafa, Ghazally I. Y., and Tian, Y., 2018b), respectively. The robustness of this strategy depends on the technique utilized to estimate the unknown dynamics, like time-delay estimation (Ahmed, S., Wang, H., and Tian, Y., 2018, Wang et al., 2016c), ultra-local model (Wang et al., 2015b, Michel et al., 2010). Unfortunately, the non-zero estimation error always remains if the estimator is not selected well. In addition, the measurement noise diminishes the performance of the closedloop control system significantly. Concerning this error, a new adaptive fuzzy based fractional order is introduced. As such, compensation can be real-time for both uncertainties and outside in the active suspension model. Moreover, the benefits of adaptive fuzzy control considerably motivate the usage in nonlinear system identification and control. Various reasons have prompted large research interests in the application of adaptive fuzzy control for control objectives over conventional control techniques (Dragomir et al., 2015, Mustafa, Ghazally I. Y., Wang, H.P., and Tian, Y., 2013). Amongst them the principal points are: first, a superior performance is obtained since the adaptive fuzzy controller can adapt itself to the various conditions. Second; by applying adaptive law, the dynamics of the plant can be learned during operation (Wang, 1993). The whole control strategy presented in this paper refers to the extended state observer (ESO) based on modelfree adaptive fuzzy logic control (MFAFLC).

The advantages of the proposed controller are that the information of the active suspension model is not needed. In this way, the complicated suspension dynamics is avoided thanks to modelfree control (MFC). Another advantage is the robustness towards the external disturbances thanks to the simultaneous application of the adaptive fuzzy logic compensator which makes possible to compensate the ESO estimation error online. Mainly the proposed controller has three components. First, the ESO is utilized to estimate the unknown uncertain dynamics via the knowledge of the active suspension system input and output signals. Second, the modelfree based iPI control is used to overcome the existing controller's complexity, insert required performances, and reduce the high order derivative output. The last one is the continuous adaptive fuzzy logic control based on fraction order control term and it is used to compensate

https://www.sic.ici.ro 
the ESO estimation error in order to guarantee the finite-time convergence and ensure the global stability of the closed-loop system. The results demonstrate that the model-free adaptive fuzzy logic control (MFAFlC) outperforms iPID and proportional-integral-derivative (PID) controllers in ride comfort and ride safety.

The paper is structured into five parts as follows. In Section 2, the problem formulation of the nonlinear half vehicle active suspension system and the system requirements are presented. The detailed design steps of the model-free adaptive fuzzy logic control are conducted and the stability analysis is proved in Section 3. Section 4 presents the simulation results to confirm the performance of MFAFLC controller compared with the iPID, classical PID and passive model. Finally, in Section 5, some concluding remarks and future work are summarized.

\section{Problem Formulation}

\subsection{Nonlinear Half-car Model}

In this section, the model of the half-car active suspension is provided in details and illustrated in Figure 1. This model has been widely used in the prior research due to its symmetry which includes the heave and pitch motions of the car body (Wang, G., Chen, C. and Yu, S., 2016a, Sun et al., 2014). In this figure, $M$ and $J$ stand for the masses of both the car body and moment of inertia for the pitch motion, respectively. $m_{t 1}$ and $m_{t 2}$ are the unsprung masses of the front and the rear tires, respectively. $z_{1}$, and $z_{2}$ are the displacements of the front, the rear car body, respectively. $a$ stands for the distance between the center of mass and the front axle, $b$ stands for the distance between the center of mass and the rear axle. $F_{d f}, F_{d r}$, $F_{s f}$, and $F_{s r}$ denote the forces provided by the springs and dampers for front and rear wheels, respectively, and $F_{t f}, F_{t r}$ are the elasticity forces of the tires. For the car body, $z_{c}$ is the vertical displacement, $\theta$ is the pitch angle, $z_{f}, z_{r}$ are the unsprung mass displacements, and $z_{01}, z_{02}$ are the road inputs to the related wheel. $u_{1}, u_{2}$ are the control signals of the active systems. The ideal dynamic equations of the half-car model can be written as follows:

$$
\left\{\begin{array}{l}
M \ddot{z}_{c}+F_{d f}+F_{d r}+F_{s f}+F_{s r}=u_{1}+u_{2} \\
I \ddot{\theta}+a\left(F_{d f}+F_{s f}\right)-b\left(F_{d r}+F_{s r}\right)=a u_{1}-b u_{2} \\
m_{t 1} \ddot{z}_{f}-F_{d f}-F_{s f}+F_{t f}=-u_{1} \\
m_{t 2} \ddot{z}_{r}-F_{d r}-F_{s r}+F_{t r}=-u_{2}
\end{array}\right.
$$

where the forces provided by the spring, the damper and the tire are described as follows:

$$
\begin{aligned}
& \left\{\begin{array}{l}
F_{s f}=k_{f 1} \Delta y_{f} \\
F_{s r}=k_{r 1} \Delta y_{r}
\end{array}\right. \\
& \left\{\begin{array}{l}
F_{d f}=b_{f} \Delta \dot{y}_{f} \\
F_{d r}=b_{r} \Delta \dot{y}_{r}
\end{array}\right. \\
& \left\{\begin{array}{l}
F_{t f}=k_{f 2}\left(z_{f}-z_{01}\right) \\
F_{d r}=k_{r 2}\left(z_{r}-z_{02}\right)
\end{array}\right.
\end{aligned}
$$

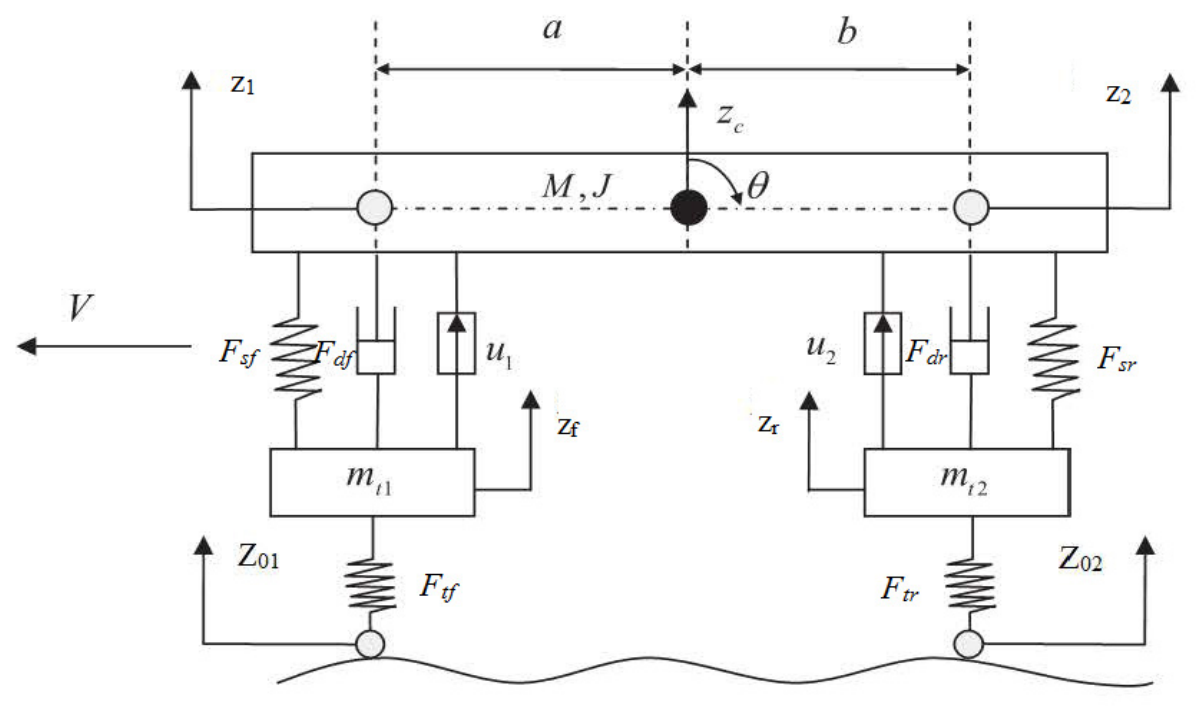

Figure 1. Half-car model with active suspension 
where $k_{f 1}$ and $k_{r 1}$ are the suspension stiffness coefficients; $b_{f}$ and $b_{r}$ are the suspension damping coefficients; $k_{f 2}$ and $k_{r 2}$ are the stiffness coefficients of the tires. $\Delta y_{f}$ and $\Delta y_{r}$ denote the front and the rear suspension deflection.

$$
\left\{\begin{array}{l}
\Delta y_{f}=z_{c}+a \sin \theta-z_{f} \\
\Delta y_{r}=z_{c}-b \sin \theta-z_{r}
\end{array}\right.
$$

By defining the state variables, $x_{1}=z_{c}+a \sin \theta-z_{f}$ is the suspension deflection of the front car body, $x_{2}=z_{c}-b \sin \theta-z_{r}$ is the suspension deflection of the rear car body, $x_{3}=z_{f}-z_{01}$ is the front tire deflection, $x_{4}=z_{r}-z_{02}$ is the rear tire deflection, $x_{5}=\dot{z}_{c}+a \theta \cos \theta$ is the vertical velocity of the front car body, $x_{6}=\dot{z}_{c}-b \theta \cos \theta$ is the vertical velocity of the rear car body, $x_{7}=\dot{z}_{f}$ is the vertical velocity of the front wheel and $x_{8}=\dot{z}_{r}$ is the vertical velocity of the rear wheel.

For the half-vehicle model active suspension form, the state vector is selected as:

$$
X=\left[\begin{array}{llllllll}
x_{1} & x_{2} & x_{3} & x_{4} & x_{5} & x_{6} & x_{7} & x_{8}
\end{array}\right]^{\mathrm{T}}
$$

The output vector can be expressed as:

$$
Y=\left[\begin{array}{llllllll}
\ddot{z}_{c} & \ddot{\theta} & z_{1}-z_{f} & z_{2}-z_{r} & F_{d y n 1} & F_{d y n 2} & u_{1} & u_{2}
\end{array}\right]^{\mathrm{T}}(7)
$$

where $F_{d y n 1}, F_{d y n 2}$ are the dynamic loads for the front and the rear wheels, respectively.

It is to be noted that with a change in the payload, there will be variation in the vehicle load, and this will correspondingly make a change in the vehicle mass $M$ and the wheels masses $m_{t 1}, m_{t 2}$. One can find various efforts that investigate into uncertain systems in the literature, see (Song, J., Niu, Y. \& Zou, Y., 2018, Sun, H., et al., 2018).

In this work, we consider that

$M_{\text {min }} \leq M \leq M_{\text {max }}, \quad\left(m_{t 1}\right)_{\text {min }} \leq m_{t 1} \leq\left(m_{t 1}\right)_{\max }$ and $\left(m_{t 2}\right)_{\min } \leq m_{t 2} \leq\left(m_{t 2}\right)_{\max }$.

\subsection{Problem Statement}

The following aspects are considered performance requirements of active suspension systems:

1. Ride comfort: As it is widely known, the main task of active suspensions control is to design a controller capable of isolating the car body from external disturbances, i.e., minimizing the vertical and the pitch acceleration of the car to ensure the ride comfort.
2. Suspension space limit: It could be noticed that the suspension is exposed to induced vibrations on the road. Thus, the maximum dynamic limits of the suspension's equilibrium position should be controlled within as follows:

$\left\{\begin{array}{l}z_{1}-z_{f}<z_{\text {f } \max } \\ z_{2}-z_{r}<z_{r \max }\end{array}\right.$

where $z_{\mathrm{f} \max }$ and $z_{\mathrm{r} \max }$ are the upper limits of suspension deflections for the both parts (front and rear), respectively.

3. Road holding: To ensure the ride safety, the wheels must come in contact with the road surface firmly and uninterrupted, while the dynamic wheels' loads should not exceed the static loads as:

$$
\left\{\begin{array}{l}
F_{d y n 1}<9.8\left(\frac{\mathrm{bM}}{\mathrm{a}+\mathrm{b}}+\mathrm{m}_{\mathrm{t} 1}\right) \\
F_{d y n 2}<9.8\left(\frac{\mathrm{aM}}{\mathrm{a}+\mathrm{b}}+\mathrm{m}_{\mathrm{t} 2}\right)
\end{array}\right.
$$

4. Actuator saturation: Considering the amplitude saturation which occurs in all the physical devices, the actuator power limit is considered as:

$$
\left\{\begin{array}{l}
\left|u_{1}\right| \leq u_{1 \max } \\
\left|u_{2}\right| \leq u_{2 \max }
\end{array}\right.
$$

\section{Model-free Adaptive Fuzzy Logic Control}

The comprehensive active vehicle suspension system using the MFAFLC is shown in Figure 2. In this part, the extended state observer (ESO), the model-free based intelligent proportional-integralderivative (iPID), and the model-free adaptive fuzzy logic control (MFAFC) are introduced.

\subsection{Model-free based Intelligent Proportional- Integral Control}

For a general unknown nonlinear model, the modelfree control is defined as (Fliess and Join, 2013):

$y^{(\mathrm{v})}(\mathrm{t})=\varepsilon(\mathrm{t})+\alpha u(\mathrm{t})$

where $y(t)$ denotes the output signal, $v$ is the derivative order which is commonly chosed as 1 or $2, \varepsilon(\mathrm{t})$ is an unknown term which is estimated through the control input $u(t)$ and output. Furthermore, $\varepsilon(\mathrm{t})$ is included the disturbances of the system, when $\alpha$ is an assumed constant. 


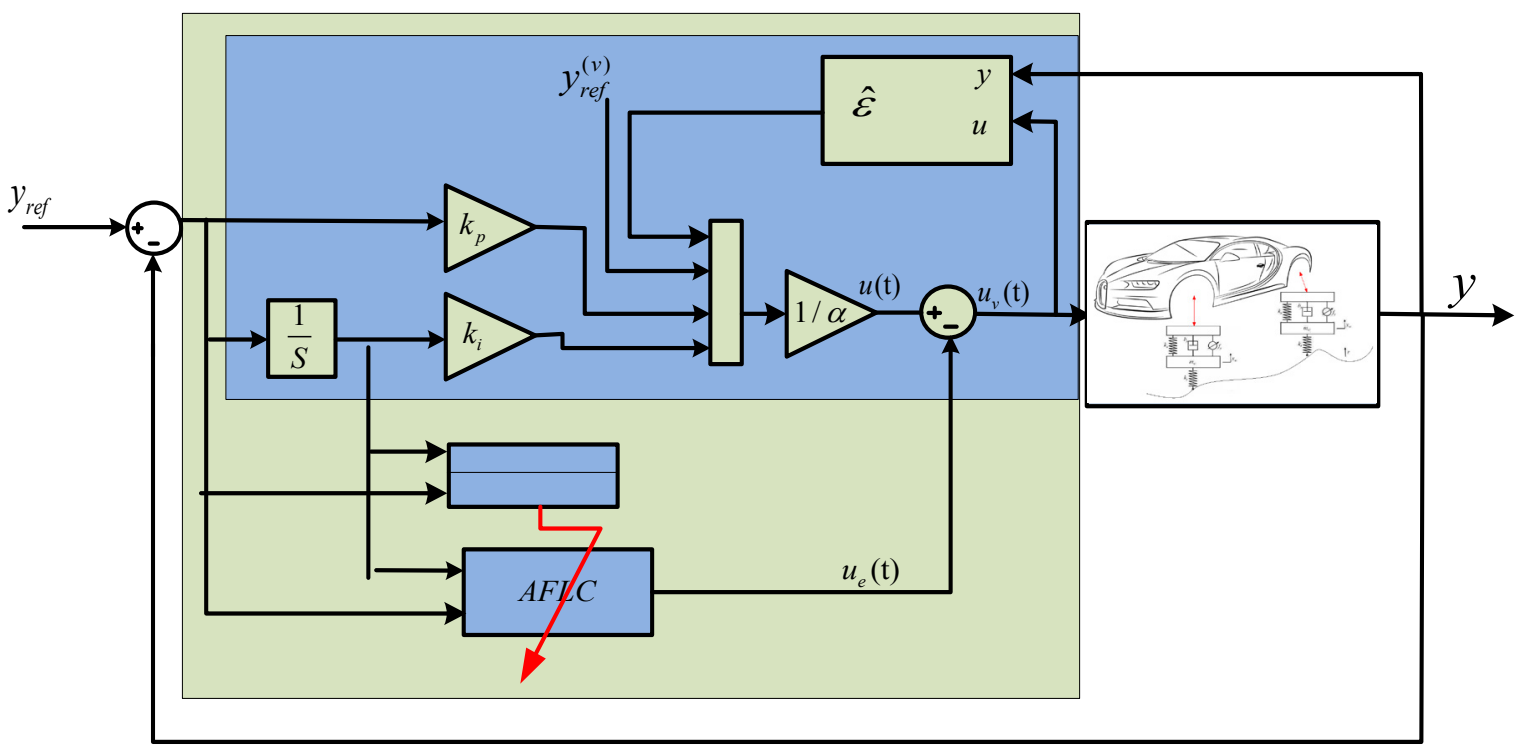

Figure 2. MFAFLC control structure

The control input of iPI is defined as:

$u(t)=\frac{1}{\alpha}\left[k_{p} e(t)+k_{i} \int e(t) d(t)+\dot{y}_{r e f}^{*}(t)-\hat{\varepsilon}(t)\right]$

where $k_{p}$ and $k_{i}$ are the corresponding classical proportional integral (PI) coefficients, $y_{r e f}^{*}(t)$ is the reference trajectory of the output, $\hat{\varepsilon}(t)$ is estimated value of $\varepsilon(t)$ and $e(t)=y^{*}{ }_{r e f}(t)-y(t)$ is the error output.

Substituting equation (11) into equation (10), the error equation can be realized as follows:

$\dot{e}(t)+k_{p} e(t)+k_{i} \int e(t) d t \approx 0$

The steady error dynamics of the closed loop is determined by the parameters $k_{p}$ and $k_{i}$ whose values can be selected according to the Hurwitz criterion and the constrained estimation error $\varepsilon(\mathrm{t})-\hat{\varepsilon}(\mathrm{t})$.

In this paper, the extended state observer (ESO) method is applied to determine $\hat{\varepsilon}(\mathrm{t})$ value which is got by the knowledge of the model input and output signals. For further comprehensive discussions on the intelligent proportional-integral (iPI) control and ESO see (Mustafa, Ghazally I. Y., Wang, H.P., and Tian, Y., 2019, Wang, H.P., Mustafa, Ghazally I. Y., and Tian, Y., 2018b).

\subsection{Model-free Adaptive Fuzzy Logic Control}

An adaptive fuzzy logic control based on the fractional order is proposed as an additional input to compensate the estimation error and measurement noise. Figure 2 shows the MFAFLC structure.

The new control input of the system is defined as:

$u_{v}(t)=u(\mathrm{t})-\alpha u_{e}(t)$

where $u_{e}(t)$ is an external adaptive fuzzy logic compensator which will be formulated later, then, the new error equation is given below:

$\dot{e}(t)+k_{p} e(t)+k_{i} \int e(t) d t=\tilde{\varepsilon}+\alpha u_{e}(t)$

where $\tilde{\varepsilon}$ is the ESO estimation error which is defined as follows:

$\tilde{\varepsilon}(\mathrm{t})=\varepsilon(\mathrm{t})-\hat{\varepsilon}(\mathrm{t})$

Now, by defining new state variables such as

$\left\{\begin{array}{l}x_{1}=\int_{0}^{t} e(t) d t \\ x_{2}=e(t)\end{array}\right.$

The state-space equations can be expressed as follows

$\left\{\begin{array}{l}\dot{x}_{1}=x_{2} \\ \dot{x}_{2}=-k_{p} x_{2}-k_{i} x_{1}+\tilde{\varepsilon}(x)+\alpha u_{e}(t)\end{array}\right.$

The error function is defined as:

$s=e+k D^{\lambda-1} \operatorname{sgn}(\mathrm{e})^{\mathrm{a}}$

where $k, a>0$ and $2>\lambda>1$.

From equation (19), if $s \rightarrow 0$, then $e \rightarrow 0$ and $\dot{e} \rightarrow 0$. 
Using the universal approximation theorem of fuzzy system, the fuzzy system $\hat{f}(x \mid \theta)$ is designed to approximate $\tilde{\varepsilon}(x)$.

Considering the inputs $x_{1}$ and $x_{2}$, the five membership MF is designed, (assume $n=2$, $i=1,2, \quad p_{1}=p_{2}=5$, and the fuzzy rules $\left(p_{1} \times p_{2}=25\right)$ can be obtained.

The fuzzy system $\hat{f}(x \mid \theta)$ is constructed by the following steps defined below:

Step1: For the variable $x_{i}(i=1,2)$, define $p_{i}$ fuzzy sets $A^{l i}(l i=1,2,3,4,5)$;

Step2: Use $\prod_{i=1}^{n} p_{i}=p_{1} \times p_{2}=25$ fuzzy rules to

construct fuzzy system $\hat{f}(x \mid \theta)$. The $\mathrm{j}^{\text {th }}$ fuzzy rule is expressed as:

$$
R^{(\mathrm{j})} \text { : if } x_{1} \text { is } A_{1}^{l_{1}} \text { and } x_{2} \text { is } A_{1}^{l_{2}} \text { then } \hat{f} \text { is } B^{l_{1} l_{2}}
$$

Then, the fuzzy inference is designed as follows:

1. Using product inference engine for the premise of fuzzy rule, $\prod_{i=1}^{2} \mu A_{i}^{l i}(x i)$ is obtained.

2. Use singleton fuzzifier to get $\bar{y}_{f}^{l_{1} l_{2}}$, where $\bar{y}_{f}^{l_{1} l_{2}}$ is the point $\left[x_{1}, x_{2}\right]$ at which $\mu_{B^{1 / 2}}\left(\bar{y}_{f}^{l_{1} l_{2}}\right)=1.0$.

3. Using product inference engine for the premise and conclusion of fuzzy rule, then $\bar{y}_{f}^{l_{1} l}\left(\prod^{2} \mu A_{i}^{l i}(x i)\right)$ is obtained, and using the union operator for different fuzzy rules, then the output of the fuzzy system can be obtained as: $\sum_{l_{1}=1}^{5} \sum_{l_{2}=1}^{5} \bar{y}_{f}^{l_{l} l_{2}}\left(\prod_{i=1}^{2} \mu_{A_{i}^{i}}\left(x_{i}\right)\right)$.

4. Using the center average defuzzifier, the output of the fuzzy system can be obtained:

$$
\hat{f}(x \mid \theta)=\frac{\sum_{l_{1}=1}^{5} \sum_{l_{2}=1}^{5} \bar{y}_{f}^{l_{1} l_{2}}\left(\prod_{i=1}^{2} \mu_{A_{i}^{i_{i}}}\left(x_{i}\right)\right)}{\sum_{l_{1}=1}^{5} \sum_{l_{2}=1}^{5}\left(\prod_{i=1}^{2} \mu_{A_{i}^{i}}\left(x_{i}\right)\right)}
$$

where $\mu_{A_{i}^{i}}\left(x_{i}\right)$ is MF of $x_{i}$.

Let $\bar{y}_{f}^{l_{f} l_{2}}$ be the freedom parameter, $\theta=\left[\bar{y}_{f}^{1} \ldots \bar{y}_{f}^{25}\right]^{\mathrm{T}}$ is a parameter vector, then, by introducing the fuzzy basis vector $\xi(x)$, equation (20) becomes:

$\hat{f}(x \mid \theta)=\hat{\theta}^{T} \xi(x)$

where $\xi(x)$ is the fuzzy basis vector with $\prod_{i=1}^{n} p_{i}=p_{1} \times p_{2}=25$ elements, its $l_{1} l_{2}^{\text {th }}$ element is $\xi_{l_{1}}(x)=\frac{\prod_{i=1}^{2} \mu_{A_{i}^{l_{i}}}\left(x_{i}\right)}{\sum_{l_{1}=1}^{5} \sum_{l_{2}=1}^{5}\left(\prod_{i=1}^{2} \mu_{A_{i}^{l_{i}}}\left(x_{i}\right)\right)}$

The optimum parameter is set as:

$\theta^{*}=\arg \min _{\theta \in \Omega}[\sup |\hat{f}(x \mid \theta)-\varepsilon(x)|]$

Then,

$\varepsilon(x)=\theta^{* T} \xi(x)+\psi$

where $\psi$ is the approximation error.

Now, substituting equation (21) from equation (24), one has the following relation:

$$
\begin{aligned}
\varepsilon(x)-\hat{f}(x) & =\theta^{* T} \xi(x)+\psi-\hat{\theta} \xi(x) \\
& =-\tilde{\theta}^{T} \xi(x)+\psi
\end{aligned}
$$

The Lyapunov function is defined as:

$V=\frac{1}{2} S^{2}+\frac{1}{2 \gamma} \tilde{\theta}^{T} \tilde{\theta}$

with $\gamma>0$ and $\tilde{\theta}=\hat{\theta}-\theta^{*}$.

Its derivative can be calculated as

$\dot{V}=s \dot{s}+\frac{1}{\gamma} \tilde{\theta}^{T} \dot{\hat{\theta}}=s\left(\dot{e}+k D^{\lambda} \operatorname{sgn}(\mathrm{e})^{\mathrm{a}}\right)+\frac{1}{\gamma} \tilde{\theta}^{T} \dot{\theta}$

Substituting $\dot{e}$ from equation (15) into the above equation, one has

$$
\begin{aligned}
\dot{V} & =s\left(-k_{p} e(t)-k_{i} \int e(t) d t+\tilde{\varepsilon}+\alpha u_{e}(\mathrm{t})\right. \\
& \left.+k D^{\lambda} \operatorname{sgn}(\mathrm{e})^{\mathrm{a}}\right)+\frac{1}{\gamma} \tilde{\theta}^{T} \dot{\hat{\theta}}
\end{aligned}
$$

The external control $u_{e}(t)$ is designed as:

$u_{e}(t)=\frac{1}{\alpha}\left(\begin{array}{l}k_{p} e(t)+k_{i} \int e(t) d t-\hat{f}(x) \\ -k D^{\lambda}\left[\operatorname{sgn}(e)^{a}\right]-\eta_{1} s-\eta_{2} \operatorname{sgn}(s)\end{array}\right)$

Then,

$$
\begin{aligned}
\dot{V} & =s\left(\tilde{\varepsilon}(x)-\hat{f}(x)-\eta_{1} s-\eta_{2} \operatorname{sgn}(\mathrm{s})\right)+\frac{1}{\gamma} \tilde{\theta}^{T} \dot{\hat{\theta}} \\
& =s\left(-\tilde{\theta}^{T} \xi(x)+\psi-\eta_{1} s-\eta_{2} \operatorname{sgn}(\mathrm{s})\right)+\frac{1}{\gamma} \tilde{\theta}^{T} \dot{\hat{\theta}} \\
& =\psi s-\eta_{1} s^{2}-\eta_{2}|s|+\tilde{\theta}^{T}\left(\frac{1}{\gamma} \dot{\hat{\theta}}-s \xi(x)\right)
\end{aligned}
$$

Taking $\eta_{2}>|\psi|_{\max }+\eta_{0} \cdot \eta_{0}>0$, the adaptive law is defined as:

$\dot{\hat{\theta}}=\gamma s \xi(x)$ 
Then,

$$
\dot{V}=\psi s-\eta_{1} s^{2}-\eta_{2}|s| \leq-\eta_{0}|s| \leq 0
$$

From the above analysis, it can be noticed that fuzzy system approximation error can be overcome by the robust term $\eta_{2} \operatorname{sgn}(\mathrm{s})$.

From equation (32), one has

$$
\int_{0}^{t} \dot{V} d t \leq-\eta_{0} \int_{0}^{t}|s| d t, \text { i.e. } V(t)-V(0) \leq-\eta_{0} \int_{0}^{t}|s| d t
$$

Since $V(0)$ is limited and $V(t)$ is non increasing and limited, $s, \dot{s}$ and $\tilde{\theta}$ are all limited, it can be concluded that $\int|s| d t$ is limited. From Barbalat Lemma (Ioannou and Sun, 1996), when $t \rightarrow \infty$, one has $s \rightarrow 0$, then $e \rightarrow 0, \dot{e} \rightarrow 0$. Finally, the MFAFLC design ensures the asymptotic stability of the system and makes the tracking error converge to zero in finite time.

\section{Simulation Results and Discussions}

In this part, the proposed control scheme is implemented to the half-car active suspension system shown in figure (2). The performances of MFAFLC are compared with the classical PID, and iPID controllers. Their corresponding numerical results are presented to confirm the effectiveness of the proposed controller.

The parameters of the half-car model are taken from (Wang, G., Chen, C. and Yu, S., 2016a) whose values are presented in Table 1 . With the sprung mass $M$ and the unsprung masses $m_{t 1}, m_{t 2}$ (front and rear), it is supposed that $M$ belongs to the range $[450 \mathrm{~kg}, 550 \mathrm{~kg}], m_{t 1}$ and $m_{t 2}$ belong to the range [ $27 \mathrm{~kg}, 33 \mathrm{~kg}$ ].

The parameters of MFAFLC controller were set as follows:

$k_{p}=18.1409, \quad k_{i}=18.1413, \quad \gamma=5000 \quad$ and $k_{d}=0.0021$.
To clarify the advantages of the MFAFLC concerning the ride comfort and ride safety the simulation tests for three different road profiles is conducted below:

Case 1: the classic bump road profile is represented as follows:

$z_{01}(\mathrm{t})=\left\{\begin{array}{lc}\frac{h}{2}\left(1-\cos \left(\frac{2 \pi \mathrm{V}}{l} \mathrm{t}\right)\right), & 0 \leq \mathrm{t} \leq \frac{1}{V} \\ 0, & \text { else }\end{array}\right.$

where $h=0.05 \mathrm{~m}$ is the bump height, $l=3 \mathrm{~m}$ is the bump length, $V=15 \mathrm{~m} / \mathrm{s}$ is the velocity of the passing vehicle, and $z_{02}(\mathrm{t})=z_{01}\left(\mathrm{t}-\frac{a+b}{V}\right)$.

Case 2: To test the performance near the system resonance frequency, the sinusoidal road profile is considered as introduced in (Pan et al., 2016) as:

$z_{01}(\mathrm{t})=0.006 \sin (2 \pi \mathrm{t})$

with $z_{02}(\mathrm{t})=z_{01}\left(\mathrm{t}-\frac{a+b}{V}\right)$

Case 3: the random profile, in this work class D road profile is selected; equation (21) represents the random road input as (Li, H., Jing, X., and Karimi, H. R., 2014a):

$\dot{x}_{g}(\mathrm{t})=-2 \pi f_{0} v x_{g}(\mathrm{t})+2 \pi n_{0} \sqrt{G_{q}\left(n_{0}\right) v} w(\mathrm{t})$

where $f_{0}$ represents the space cutoff frequency of road, $v$ represents vehicle speed, $n_{0}$ is the spatial reference frequency, $G_{q}\left(n_{0}\right)$ is the power spectral density of road profiles, and $w(\mathrm{t})$ is the input white noise. Figure 7 illustrates the random road input.

\subsection{The Bump Road Results and Discussions}

The dynamic responses of the car vertical and angular accelerations, using passive model, classical PID, iPID and MFAFFC controllers are shown in Figures 3 and 4, while the front and the rear responses of the suspension deflections, the dynamic wheel loads, and inputs force using the proposed method are shown in Figures 5-7.

Table 1. The parameters of the half-car model

\begin{tabular}{cccccc}
\hline$J$ & $a$ & $b$ & $k_{f 1}$ & $k_{r 2}$ & $k_{f 2}$ \\
$910 \mathrm{~kg} \mathrm{~m} 2$ & $1.5 \mathrm{~m}$ & $1.5 \mathrm{~m}$ & $10000 \mathrm{~N} / \mathrm{m}$ & $10000 \mathrm{~N} / \mathrm{m}$ & $100000 \mathrm{~N} / \mathrm{m}$ \\
\hline$k_{r 2}$ & $b_{f}$ & $b_{r}$ & $Z_{1 \max }$ & $Z_{2 \max }$ & $u_{1,2 \max }$ \\
$100000 \mathrm{~N} / \mathrm{m}$ & $1000 \mathrm{~N} \mathrm{~s} / \mathrm{m}$ & $1000 \mathrm{~N} \mathrm{~s} / \mathrm{m}$ & $0.1 \mathrm{~m}$ & $0.1 \mathrm{~m}$ & $2500 \mathrm{~N}$ \\
\hline
\end{tabular}


Figures 3 and 4 show the vertical and the angular body acceleration responses; it is clearly noticed that the MFAFLC has lower amplitude and smaller settling time response in comparison with others controllers, which implies that the proposed method achieves significantly better performance on ride comfort over the others controllers.

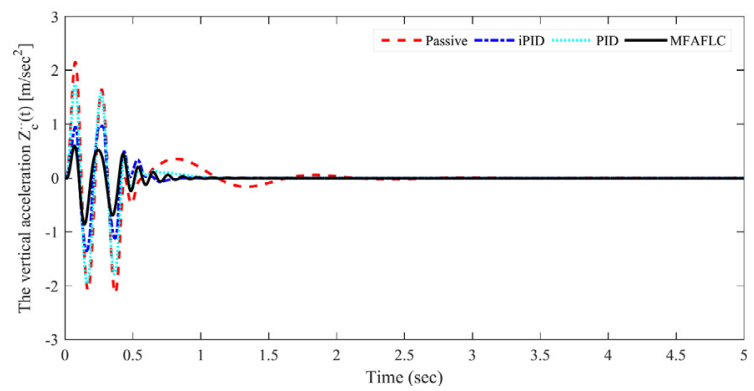

Figure 3. The vertical body acceleration response

The suspension deflections measured in Meters, the dynamic wheels load ratios and the force inputs responses measured in Newtons are shown in Figures 5-7, respectively. Also, from these figures it can be noticed that all the achieved results have values which are lower than the permissible limits as mentioned above in the suspension performances requirements.

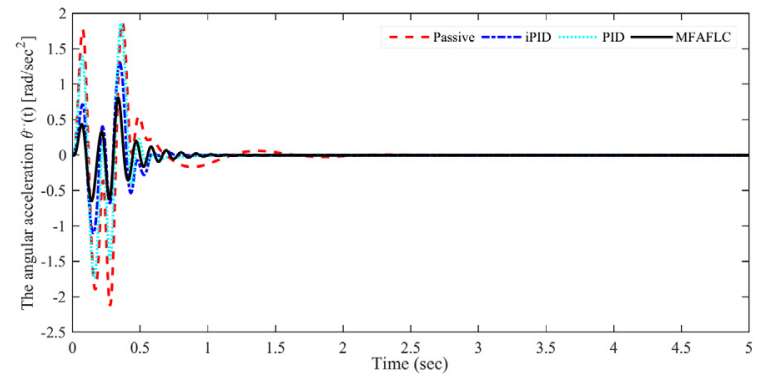

Figure 4. The angular body acceleration response

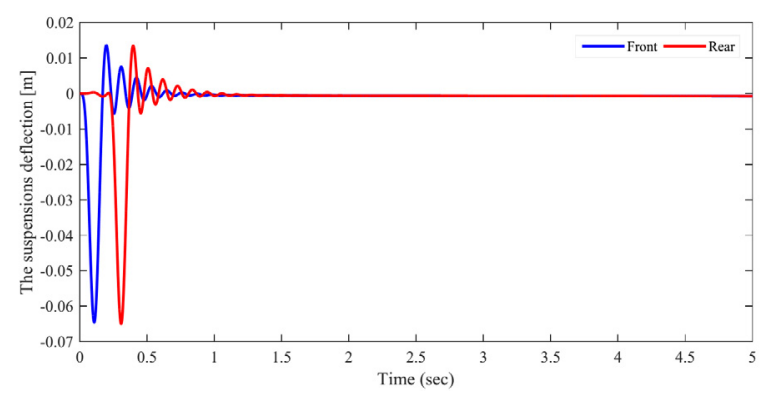

Figure 5. The suspension deflections

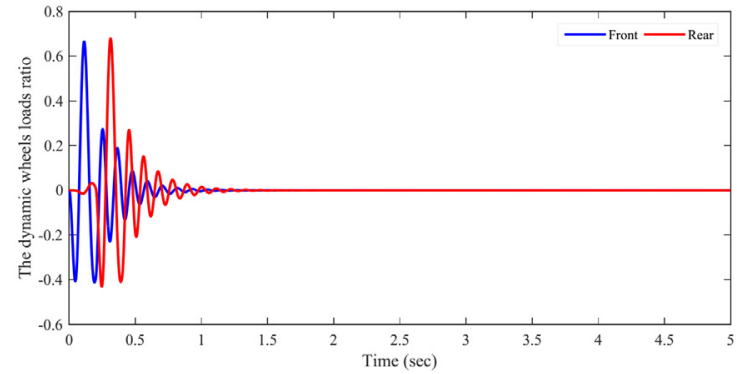

Figure 6. The dynamic wheels load ratios

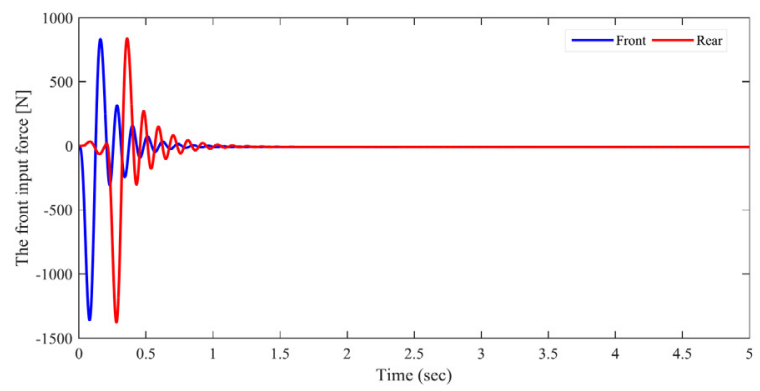

Figure 7. The response of the input forces

\subsection{The Sinusoidal Road Profile}

For a vehicle which passes over the sinusoidal road excitation, the dynamic responses of vertical and angular accelerations, using passive model, classical PID, iPID and MFAFFC controllers are shown in Figures 8-9. Numerically, the maximum values of body angular acceleration are $0.5478 \mathrm{rad} / \mathrm{sec}^{2}$ with proposed MFAFLC controller, $0.862 \mathrm{rad} / \mathrm{sec}^{2}$ with iPID, 1.4373 $\mathrm{rad} / \mathrm{sec}^{2}$ with $\mathrm{PID}$, and $1.7193 \mathrm{rad} / \mathrm{sec}^{2}$ with passive system. Furthermore, the MFAFLC diminishes the overshoot value of the vertical body acceleration by $61.1 \%$ in comparison with the passive system, while reaching $54.23 \%$ and $33.15 \%$ with respect to PID and iPID. It can be remarked that the amplitude of the body vertical and angular accelerations diminishes much faster in the proposed controller when compared with the other controllers; this will ensure much better ride comfort.

Figures 10-12 show the dynamic responses of the suspension deflections, the dynamic wheel's load ratios, and the force inputs, respectively. From these figures it can be noticed that from these figures, all the obtained results have values which are smaller than the permissible boundaries as mentioned above in the suspension performances' conditions.

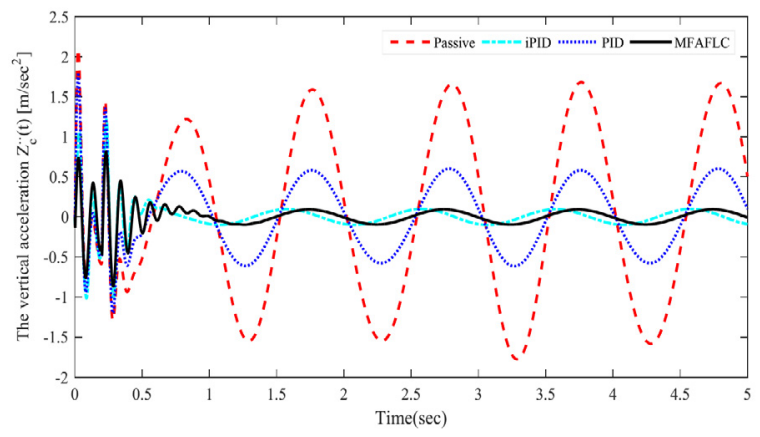

Figure 8. The vertical body acceleration response

https://www.sic.ici.ro 


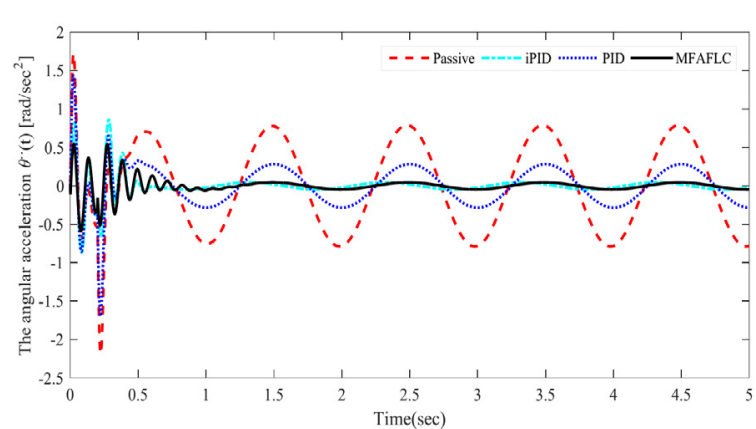

Figure 9. The angular body acceleration response

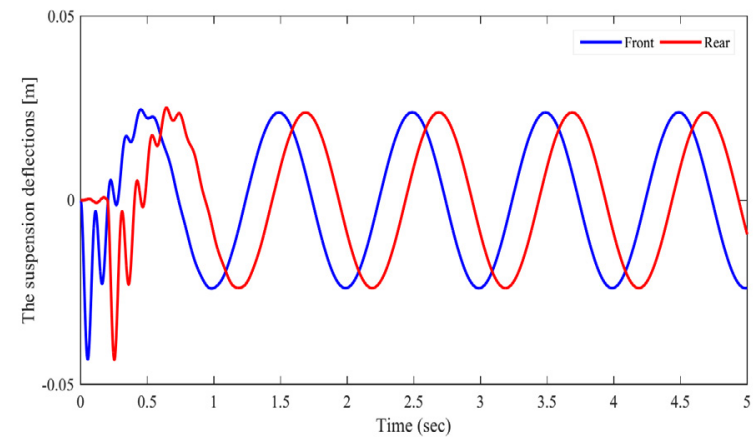

Figure 10. The suspension deflections

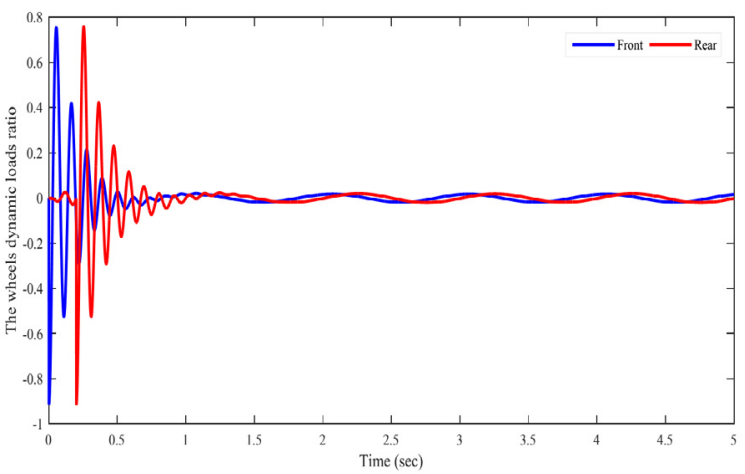

Figure 11. The dynamic wheels load ratios

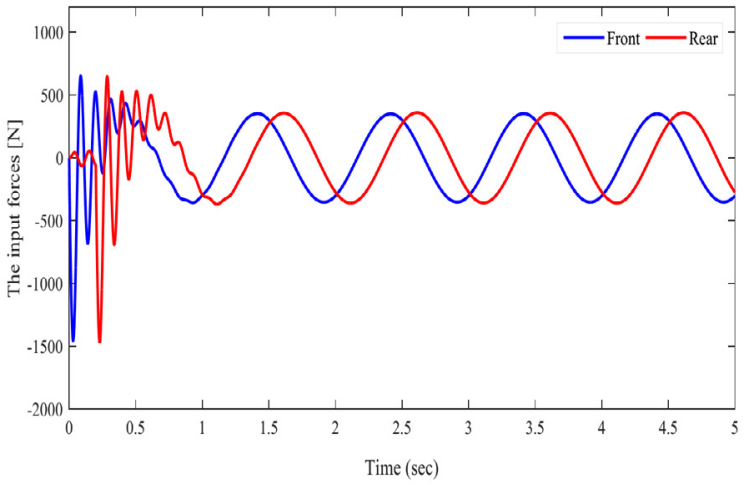

Figure 12. The response of the input forces

\subsection{The Random Road Results and Discussions}

To improve the quality of the ride, it is important to separate the car body from road excitements and to reduce the peak resonance of the vehicle body mass close to $1 \mathrm{~Hz}$ which has been identified to be a sensitive response to the human body (Metered, H., Bonello, P., and Oyadiji, S., 2010, Shehata, A., Metered, H., and Oraby, W. A., 2015). In Figures 13-14 the Fast Fourier Transform modulus (FFT) is presented for both vertical and angular body accelerations behaviors. The proposed controller shows the lowest resonance peaks in comparison with other controllers.

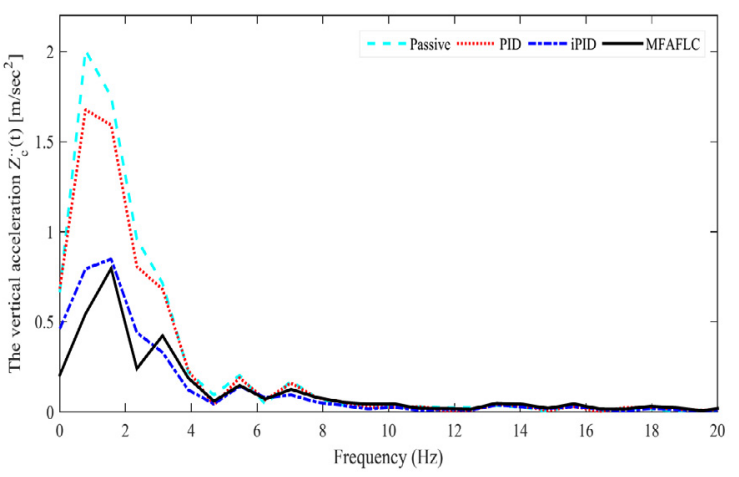

Figure 13. The vertical body acceleration response

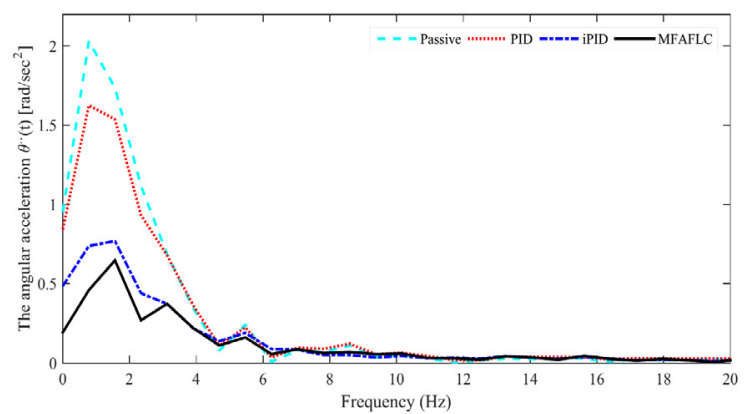

Figure 14. The angular body acceleration response

The results of the present analysis confirm that the MFAFLC strategy gives significant performance enhancements for the comfort of the ride and also for the ride safety. The proposed controller achieved a good result which keeps the boundaries of the dynamic responses of the suspension deflections, the dynamic wheel's load, and the force inputs for all the road profiles are taken into consideration.

\section{Conclusion and Future Work}

In this paper, the robust model-free adaptive fuzzy logic control based on the fractional order and the extended state observer for half-car active suspension systems meant to enhance the ride comfort and keep acceptable boundaries for the suspensions deflections, the dynamic wheels loads, and input forces are presented. The model with parameter variation and three types of road profiles have been considered in this work. To confirm the advantage of the proposed controller 
the simulation results were analyzed and compared with iPID and classical PID controllers.

It can be concluded that the proposed controller improved the riding comfort when compared with other approaches and kept acceptable limitations for the suspension deflections, the road holding and actuator saturation. Moreover, experimental verification of the model-free adaptive fuzzy logic control could be considered an immersive starting point in future work.

\section{REFERENCES}

1. Ahmed, S., Wang, H. \& Tian, Y. (2018). Model-free control using time delay estimation and fractional-order nonsingular fast terminal sliding mode for uncertain lower-limb exoskeleton, Journal of Vibration and Control, 24(22), 5273-5290.

2. Cao, J., Li, P. \& Liu, H. (2010). An interval fuzzy controller for vehicle active suspension systems, IEEE Transactions on Intelligent Transportation Systems, 11, 885-895.

3. Cao, J., Liu, H., Li, P. \& Brown, D. J. (2008). State of the art in vehicle active suspension adaptive control systems based on intelligent methodologies, IEEE transactions on intelligent transportation systems, 9(3), 392-405.

4. Chen, H. \& Huang, S. (2005). Adaptive control for active suspension system. In International Conference on Control and Automatic, June, Budapest, Hungary.

5. Chen, M. Z., Hu, Y., Li, C. \& Chen, G. (2015). Performance benefits of using inerter in semiactive suspensions, IEEE Transactions on Control Systems Technology, 23(4), 1571-1577.

6. Chen, S., He, R., Liu, H. \& Yao, M. (2012). Probe into necessity of active suspension based on LQG control, Physics Procedia, 25, 932-938.

7. Demir, O., Keskin, I. \& Cetin, S. (2012). Modeling and control of a nonlinear halfvehicle suspension system: a hybrid fuzzy logic approach, Nonlinear Dynamics, 67(3), 2139-2151.

\section{Acknowledgments}

This work was partially supported by the National Natural Science Foundation of China (61773212), by the Natural Science Foundation of Jiangsu Province (BK20170094), by International Science \& Technology Cooperation Program of China (2015DFA017 10), and by the 11th Jiangsu Province Six talent peaks of high-level talents (2014_ZBZZ_005).

8. Dragomir, O., Dragomir, F., Stefan, V. \& Minca, E. (2015). Adaptive NeuroFuzzy Inference Systems-An Alternative Forecasting Tool for Prosumers, Studies in Informatics and Control, 24(2), 351-360, DOI: $10.24846 / \mathrm{v} 24 \mathrm{i} 3 \mathrm{y} 201511$

9. Fliess, M. \& Join, C. (2008). Intelligent PID controllers. In 16th Mediterrean Conference on Control and Automation, (pp. 326-331).

10. Fliess, M. \& Join, C. (2013). Model-free control, International Journal of Control, 86(12), 2228-2252.

11. Hafaifa, A., Laaouad, F. \& Laroussi, K. (2010). Fuzzy approach applied in fault detection and isolation to the compression system control, Studies in Informatics and Control, 19(1), 17, DOI: 10.24846/ v19i1y201002

12. Han, S., Wang, H. P. \& Tian, Y. (2018). Model-free based adaptive nonsingular fast terminal sliding mode control with time-delay estimation for a 12 DOF multi-functional lower limb exoskeleton, Advances in Engineering Software, 119, 38-47.

13. Hrovat, D. (1997). Survey of advanced suspension developments and related optimal control applications 1, 2, Automatica, 33(10), 1781-1817.

14. Ioannou, P. A. \& Sun, J. (1996). Robust adaptive control, vol. 1. PTR Prentice-Hall Upper Saddle River, NJ. 
15. Li, H., Jing, X. \& Karimi, H. R. (2014). Output-feedback-based $H_{\infty}$ control for vehicle suspension systems with control delay, IEEE Transactions on Industrial Electronics, 61, 436-446.

16. Li, H., Yu, J., Hilton, C. \& Liu, H. (2013). Adaptive sliding-mode control for nonlinear active suspension vehicle systems using T-S fuzzy approach, IEEE Transactions on Industrial Electronics, 60(8), 3328-3338.

17. Li, P., Lam, J. \& Cheung, K. C. (2014a). Multi-objective control for active vehicle suspension with wheelbase preview, Journal of Sound and Vibration, 333(21), 5269-5282.

18. Li, P., Lam, J. \& Cheung, K. C. (2014b). Velocity-dependent multi-objective control of vehicle suspension with preview measurements, Mechatronics, 24(5), 464-475.

19. Li, S., Wang, H. P., Tian, Y., Aitouch, A. \& Klein, J. (2016). Direct power control of DFIG wind turbine systems based on an intelligent proportional-integral sliding mode control, ISA transactions, 64, 431-439.

20. Metered, H., Bonello, P. \& Oyadiji, S. (2010). An investigation into the use of neural networks for the semi-active control of a magnetorheologically damped vehicle suspension. In Proceedings of the Institution of Mechanical Engineers, Part D: Journal of Automobile Engineering, vol. 224 (pp. 829-848).

21. Michel, L., Join, C., Fliess, M., Sicard, P. \& Chériti, A. (2010). Model-free control of $\mathrm{dc} / \mathrm{dc}$ converters. In IEEE 12th Workshop on Control and Modeling for Power Electronics (COMPEL) (pp. 1-8).

22. Moradi, M. \& Fekih, A. (2014). Adaptive PID-sliding-mode fault-tolerant control approach for vehicle suspension systems subject to actuator faults, IEEE Transactions on Vehicular Technology, 63(3), 1041-1054.

23. Mustafa, Ghazally I. Y., Ali, A. T., Bashier, E. \& Elrahman, M. F. (2013). Neuro-fuzzy controller design for a dc motor drive, University of Khartoum Engineering Journal, 3(1), 7-11.
24. Mustafa, Ghazally I. Y., Wang, H. P. \& Tian, Y. (2019). Vibration control of an active vehicle suspension systems using optimized model-free fuzzy logic controller based on time delay estimation, Advances in Engineering Software, 127(1), 141-149.

25. Pan, H., Sun, H. W., Gao, H. \& Jing, X. (2016). Disturbance observer-based adaptive tracking control with actuator saturation and its application, IEEE Transactions on Automation Science and Engineering, 13(2), 868-875.

26. Pan, H., Sun, W., Gao, H. \& Jing, X. (2017). Disturbance observer-based adaptive tracking control with actuator saturation and its application, IEEE Transactions on Automation Science and Engineering, 13, 868-875.

27. Pang, H., Zhang, X. \& Xu, Z. (2018). Adaptive backstepping-based tracking control design for nonlinear active suspension system with parameter uncertainties and safety constraints, ISA Transactions, DOI:10.1016/j. isatra.2018.11.047

28. Pusadkar, U. S., Chaudhari, S. D., Shendge, P. D. \& Phadke, S. B. (2019). Linear disturbance observer based sliding mode control for active suspension systems with non-ideal actuator, Journal of Sound and Vibration, 442, 428-444.

29. Rao, M. \& Prahlad, V. (1997). A tunable fuzzy logic controller for vehicle-active suspension systems, Fuzzy sets and systems, 85(1), 11-21.

30. Sam, Y. M., Osman, J. H. S. \& Ghani, M. R. A. (2004). A class of proportional-integral sliding mode control with application to active suspension system, Systems \& Control Letters, 51(3), 217-223.

31. Savaresi, S. M., Poussot-Vassal, C., Spelta, C., Sename, O. \& Dugard, L. (2010). Semi-active suspension control design for vehicles. Elsevier.

32. Shehata, A., Metered, H. \& Oraby, W. A. (2015). Vibration control of active vehicle suspension system using fuzzy logic controller, Vibration Engineering and Technology of Machinery, 389-399. Springer. 
33. Song, J., Niu, Y. \& Zou, Y. (2018). A Parameter-Dependent Sliding Mode Approach for Finite-Time Bounded Control of Uncertain Stochastic Systems With Randomly Varying Actuator Faults and Its Application to a Parallel Active Suspension System, IEEE Transactions on Industrial Electronics, 65(10), 8124-8132.

34. Sun, H., Li, Y., Xu, K. \& Tong, S. (2018). Fuzzy Adaptive Backstepping Control for a Class of Active Suspension Systems, IFAC-PapersOnLine, 51(31), 136-141.

35. Sun, W., Pan, H., Zhang, Y. \& Gao, H., (2014). Multi-objective control for uncertain nonlinear active suspension systems, Mechatronics, 24(4), 318-327.

36. Wang, G., Chadli, M., Chen, H. \& Zhou, Z. (2018). Event-triggered control for active vehicle suspension systems with networkinduced delays, Journal of the Franklin Institute, 356(1), 147-172.

37. Wang, G., Chen, C. \& Yu, S. (2016). Optimization and static output-feedback control for half-car active suspensions with constrained information, Journal of Sound and Vibration, 378, 1-13.

38. Wang, H. P., Li, S., Tian, Y. \& Aitouche, A. (2016). Intelligent proportional differential neural network control for unknown nonlinear system, Studies in Informaics and Control, 25(4), 445-452, DOI: 10.24846/ v25i4y 201605

39. Wang, H. P., Mustafa, Ghazally I. Y. \& Tian, Y. (2018). Model-free fractionalorder sliding mode control for an active vehicle suspension system, Advances in Engineering Software, 115(1), 452-461.

40. Wang, H. P., Tian, Y., Ni, S. \& Christov, N. (2015). Intelligent proportional trajectory tracking controllers: Using ultra-local model and time delay estimation techniques. In 27th Chinese Control and Decision Conference (CCDC) (pp. 430-435).
41. Wang, H.P., Ye, X., Tian, Y., Zheng, G. \& Christov, N. (2016). Model-free-based terminal SMC of quadrotor attitude and position, IEEE Transactions on Aerospace and Electronic Systems, 52(5), 2519-2528.

42. Wang, L.-X. (1993). Stable adaptive fuzzy control of nonlinear systems, IEEE Transactions on fuzzy systems, 1(2), 146-155.

43. Wen, S., Chen, M. Z., Zeng, Z., Yu, X. \& Huang, T. (2017). Fuzzy control for uncertain vehicle active suspension systems via dynamic sliding-mode approach, IEEE Transactions on Systems, Man, and Cybernetics: Systems, 47(1), 24-32.

44. Yagiz, N. \& Hacioglu, Y. (2008). Backstepping control of a vehicle with active suspensions, Control Engineering Practice, 16(12), 1457-1467.

45. Yoshimura, T., Kume, A., Kurimoto, M. \& Hino, J. (2001). Construction of an active suspension system of a quarter car model using the concept of sliding mode control, Journal of Sound and Vibration, 239(2), 187-199.

46. Zhang, X., Wang, H., Tian, Y., Peyrodie, L. \& Wang, X. (2018). Model-free based neural network control with time-delay estimation for lower extremity exoskeleton, Neurocomputing, 272, 178-188.

47. Zhao, F., Dong, M., Qin, Y., Gu, L. \& Guan, J. (2015). Adaptive neural-sliding mode control of active suspension system for camera stabilization, Shock and Vibration, 8, DOI: $10.1155 / 2015 / 542364$ 